Le point sur...

\title{
Angleterre : The Primary Review, un état des lieux de l'enseignement primaire
}

Maroussia Raveaud

\section{OpenEdition}

\section{Journals}

Édition électronique

URL : http://journals.openedition.org/ries/79

DOI : $10.4000 /$ ries.79

ISSN : 2261-4265

Éditeur

Centre international d'études pédagogiques

Édition imprimée

Date de publication : 1 septembre 2007

Pagination : 14-16

ISBN : 978-2-85420-570-1

ISSN : 1254-4590

Référence électronique

Maroussia Raveaud, "Angleterre : The Primary Review, un état des lieux de l'enseignement primaire », Revue internationale d'éducation de Sèvres [En ligne], 45 | septembre 2007, mis en ligne le 22 juin 2011 , consulté le 19 avril 2019. URL : http://journals.openedition.org/ries/79 ; DOI : 10.4000/ries.79

Ce document a été généré automatiquement le 19 avril 2019

(c) Tous droits réservés 


\title{
Angleterre: The Primary Review, un état des lieux de l'enseignement primaire
}

\author{
Maroussia Raveaud
}

1 L'Angleterre a une longue tradition de commissions d'enquête dressant l'état des lieux du système éducatif. Concernant l'école primaire, la dernière datait de 1967 avec le célèbre rapport Plowden ${ }^{1}$, considéré comme la bible d'une génération d'enseignants, qui consacrait les pédagogies nouvelles et les méthodes actives centrées sur l'enfant.

Quarante ans plus tard, c'est dans un contexte très différent que l'exercice est réitéré par une commission indépendante basée à l'Université de Cambridge, sous la direction de Robin Alexander, expert de l'enseignement primaire et universitaire de renom connu pour ses travaux nationaux et comparatifs ${ }^{2}$ sur l'École. Depuis la grande réforme éducative de 1988, des programmes centralisés ont été introduits, assortis de tests nationaux dont dépendent partiellement les financements, et qui servent à classer les écoles dans des palmarès hautement médiatisés. Diverses études ont montré à quel point cette nouvelle politique éducative entrait initialement en tension avec les pratiques et convictions enseignantes. Aujourd'hui, près de vingt ans après le changement de cap impulsé par le haut, un nouvel état des lieux est donc en cours.

3 La Primary Review ${ }^{3}$ a débuté en octobre 2006 par une consultation publique qui s'est faite par plusieurs voies: appel aux témoignages ouvert à tous, entretiens individuels et collectifs, recherche documentaire, auditions d'experts nationaux et étrangers. La phase de consultation s'est achevée en mars 2007 (bien que la commission accepte toujours les témoignages), et la commission procède actuellement à l'analyse des données en vue de produire les rapports intérimaires et finaux.

4 Au cœur de l'entreprise se trouve une consultation publique dont la perspective se veut large : plutôt que de se centrer d'emblée et exclusivement sur l'éducation scolaire, elle part des enfants et de l'enfance, puis s'élargit à la culture, à la société à la mondialisation 
pour s'interroger sur leurs effets respectifs sur la vie des enfants d'aujourd'hui, sur la possibilité de formuler un projet consensuel de «bonne société », et sur le rôle de l'école en la matière. Ce n'est qu'après ces ouvertures qu'arrive l'éducation en tant que telle, avec dix thèmes proposés à la réflexion publique :

Missions et valeurs : à quoi sert l'école primaire? Quelles valeurs essentielles et quels principes doit-elle promouvoir?

6 Enseignement et apprentissage: que savons-nous de la façon dont apprend et se développe un enfant? Quelle est l'influence sur ses apprentissages de son état émotionnel, de son état de santé, de ses circonstances personnelles et sociales, d'être un garçon ou une fille? Quelles sont les méthodes pédagogiques les plus efficaces à chaque âge et comment les améliorer?

7 Programmes et évaluation: qu'apprennent les enfants? Que devraient-ils apprendre? Qu'est-ce qu'un programme primaire pertinent et équilibré ? Comment et quoi évaluer, par qui, pour qui, pour quoi?

Qualité et résultats : que vaut l'éducation primaire en Angleterre? Le niveau monte-t-il ou baisse-t-il? Qu'en est-il par rapport aux autres pays? Les évaluations sont-elles fiables ? Leur utilisation est-elle adéquate?

Diversité et inclusion: les écoles primaires prennent-elles en compte de façon juste et efficace la diversité des besoins scolaires des enfants et de leurs origines culturelles? Les enfants ont-ils un accès égal à une éducation primaire de qualité ? Comment un système national d'enseignement peut-il répondre au mieux à la diversité des cultures, croyances religieuses, langues et aspirations qui caractérisent la Grande-Bretagne d'aujourd'hui ? Qu'est-ce qui constitue l'identité dans une culture plurielle, et quel doit être le rôle de l'école dans sa promotion?

10 Acteurs et locaux : comment les écoles sont-elles organisées, équipées, dirigées? Qu'en est-il de la formation initiale et de la formation continue des enseignants? Comment déployer au mieux les ressources?

11 Parents et éducation: de quelles conditions parentales le bien-être des enfants et leur réussite primaire dépendent-ils? En quoi les services éducatifs et sociaux doivent-ils aider les parents? Quels sont les enjeux de la relation école-famille?

Hors de l'école : que savons-nous de la vie des enfants hors de l'école et de l'incidence sur leur développement des diverses influences qu'ils rencontrent (famille, pairs, communauté locale, médias, etc.) ? Comment se répartissent les responsabilités entre les personnes et les institutions chargées de l'éducation, du suivi social, sanitaire et psychologique des enfants?

13 Structures et cycles: comment s'organisent et s'articulent les différentes phases et structures des institutions éducatives - accueil de la petite enfance, répartition par cycles (key stages) à l'école primaire? Y a-t-il des problèmes de cohérence, de transition et de continuité au sein des cycles et entre eux? Comment les enfants sont-ils répartis et regroupés, selon quels critères et à quelles fins? Existe-t-il des alternatives plus efficaces?

14 Financement et gouvernance : le financement des établissements est-il adéquat? Quel équilibre rechercher entre acteurs (gouvernement national, autorités locales, communauté locale, établissements)? Quel devrait être le statut des écoles confessionnelles? 
15 Alors qu'on reproche fréquemment à la législation nationale une orientation trop étroitement déterminée par l'obsession de la "rentabilité » et par des préoccupations d'ordre économique, la consultation Primary Review se caractérise par sa volonté de couvrir le plus large spectre d'enjeux possible. Est proposé comme élément de réflexion pour cet état des lieux tout ce qui peut avoir une incidence sur les apprentissages, depuis l'évolution des modèles familiaux jusqu'aux dernières découvertes des neurosciences, en passant par les modes de financement, la qualité des ressources pédagogiques ou encore l'état émotionnel de l'enfant. Certes, les thèmes de consultation ne préjugent pas du contenu du rapport final, qui dépendra des témoignages recueillis. Pour autant, ils reflètent les grands enjeux sociaux du moment et une redéfinition de la mission de l'École qui conduit à un entrelacement de thématiques relevant d'ères et de conceptions pédagogiques diverses.

Parmi les enjeux sociétaux, on trouve des échos du grand débat concernant le «multiculturalisme»: dans un pays qui refuse de formuler la question des «minorités ethniques » en termes "d'intégration ", quel degré de reconnaissance ou de valorisation accorder aux cultures minoritaires? Jusqu'où et comment l'école doit-elle s'en charger? Quelle identité collective - anglaise ou britannique - peut-elle coexister avec la promotion de la diversité et de la pluralité ?

17 Le regard posé sur l'école primaire et sur l'écolier suggère, quant à lui, un paysage éducatif en pleine redéfinition suite à deux décennies de réformes touchant aussi bien aux méthodes qu'aux missions de l'École. L'héritage de l'ère plowdenienne se fait par exemple ressentir dans une approche qui conduit à prendre en compte l'enfant sous toutes ses facettes (the whole-child) et non le seul élève, et à proposer des pédagogies fondées sur la différenciation. Mais les affirmations du rapport Plowden se sont transformées en points d'interrogation, la question étant désormais posée de délimiter les «facettes» et les facteurs de différence individuelle et collective qu'il est légitime, pertinent, possible et efficace de prendre en compte à l'école.

18 Les thèmes de consultation reflètent les priorités politiques des dernières années, mais avec des formulations permettant d'aller au-delà du simple référendum, et proposant des pistes de réflexion critique orientée vers l'avenir. Ainsi quand les tests nationaux et les classifications internationales sont évoqués, c'est pour demander à quelles conditions et dans quels buts leur emploi est susceptible d'avoir une incidence positive.

Sous l'égide de Robin Alexander, on peut s'attendre à ce que les conclusions de la Primary Review soient à la hauteur des ambitions de cette entreprise de grande envergure.

\section{NOTES}

1. Central Advisory Council for Education, Children and their Primary Schools, London, HMSO, 1967.

2. Robin Alexander, Culture and Pedagogy, Oxford, Blackwell, 2000.

3. Pour plus de détails, se reporter au site Internet de la Primary Review: http:// www.primaryreview.org.uk 
INDEX

Mots-clés : enseignement primaire, réforme

Index géographique : Angleterre, Royaume-Uni

\section{AUTEUR}

\section{MAROUSSIA RAVEAUD}

Maître de conférences à l'Université du Maine ; chercheur associé à l'Observatoire sociologique du changement, CNRS/Institut d'études politiques de Paris, France. 